\title{
Link-centric Probabilistic Coverage Model for Transceiver-free Object Detection in Wireless Networks
}

\author{
Dian Zhang, Yunhuai Liu and Lionel M. Ni \\ Department of Computer Science and Engineering, \\ Hong Kong University of Science and Technology \\ \{zhangd, yunhuai,ni\}@cse.ust.hk
}

\begin{abstract}
Sensing coverage is essential for most applications in wireless networks. In traditional coverage problem study, the disk coverage model has been widely applied because of its simplicity. Though notable recent works point out that the disk model has many critical limitations when applied in practice, few successful works have been conducted to comprehensively study the issue. Motivated by this, in this paper we propose a new coverage model called $T-R$ model. T-R model is derived from a real application of transceiver-free object detection. Compared with the traditional disk model, T-R model is able to describe many new coverage features such as the probabilistic coverage, the link-centric coverage units and the correlations between multiple coverage units. These new capabilities make T-R model a better abstraction of individual sensors. To evaluate the performance of $T-R$ model, we conduct comprehensive empirical studies based on a test-bed of 30 telosB nodes. Experimental results show that the T$R$ model can adequately describe the sensing behavior in the transceiver-free object detection applications. The average error between the model and the reality is only 8\%. Moreover, T-R model presents attractive flexibility, making it more appropriate for general coverage problem studies than the transceiver-free object detection.
\end{abstract}

\section{INTRODUCTION}

Sensing coverage is essential for most applications in wireless sensor networks [1]. In typical applications, each sensor is assumed to sense a certain area and the sensing coverage problem is to guarantee a kind of coverage while minimizing the number of nodes in use. According to different requirements on the coverage, various coverage problems have been studied such as the blanket coverage [2] [3], barrier coverage [4], surface coverage [5] and trap coverage [6].

In all these studies, a fundamental assumption is of the sensing coverage capability of individual nodes, namely the coverage model. Most of the existing works are based on the disk coverage model (also referred as disk model) [1] [2] [3] [8]. It assumes that the sensing coverage of a node is a disk, which is centered at the node and bounded by a predefined sensing range. All events and target object within the range can always be sensed. For instance, in a terrain vehicle detection application [16] the sensing range is assumed to be $55 \mathrm{~m}$. Different nodes may have different sensing ranges, leading to different sizes of the disks. And some extension works assumes that a node may have different sensing coverage ranges along different directions (e.g., [17] [18] [9] [15]), while the extension shares the core ideas with the isotropic one.

The disk model is quit successful in simplifying the sensing coverage behavior of sensor nodes and therefore is widely adopted. It, however, presents many critical limitations in real applications. Few real-world sensors have the sensing characteristics as described by the disk model. For instances, in temperature data gathering applications [19], the sensing readings are for individual points rather than a field of proximity. Noticing such gaps, a recent work [10] applied a probabilistic model to abstract the sensing behavior. It assumed the probabilistic model was available but did not mention how to obtain this probabilistic model.

In order to fill the gap between the traditional disk model and the real-world sensing behaviors, in this work we systematically investigate the characteristics of sensors in a real application and propose a novel coverage model called $T-R$ model. T-R model is derived from the transceiver-free (device-free) object detection [21] [22] [23]. Transceiver-free object detection has drawn an increasing attention in recent years. It uses the disturbance of the object to the radio environment to estimate and locate the target object. This target object does not require to carry any device. Device-free object detection is particularly suitable for security, surveillance and location-aware related applications.

Figure 1 gives an illustration of the traditional disk model and the real sensing in the transceiver-free object detection. In this three-node scenario, by disk model the coverage units are three independent and fixed-size disks (left sub-figure of Fig.1). The practice (right subfigure of Fig.1) is that there are six possible coverage 


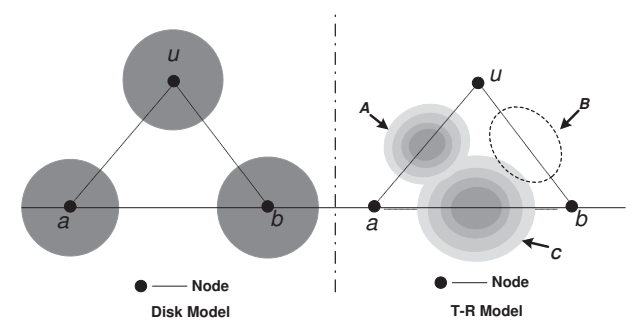

Fig. 1. Comparison between disk model and T-R model

units. These coverage units are corresponding to the six wireless links between the three nodes $u, a$ and $b$ (notice that links are directional and asymmetric in practice). Each time only two of the six can co-exist. Consider three coverage units $A:\{u, a\}, B:\{u, b\}$ and $C:\{a, b\}$. Units $A$ and $B$ can co-exist as both of them are from a single sender $u$ to different receivers $a$ and $b$. Unit $C$ is exclusive with $A$ and $B$ due to the conflicted transmitter or receiver. Moreover, the sensing contains errors, making it a probability that whether a present object can be detected or not.

To appropriately abstract such sensing coverage behaviors in these applications, T-R model includes many new features compared with the traditional disk model. The first feature is the probabilistic coverage, i.e., when an object/event is in presence, with certain probability it can be detected or sensed. Accordingly, it is also a probability that whether a field of interest is covered or not. Second, the coverage is non-node centric (in transceiver-free object tracking, it is link centric). Third, different coverage units may have correlations such as the interference and exclusive, making the traditional coverage problems more challenging. Indeed, T-R model is a more general model that most of the existing ones can be considered as its special case. For instance, disk model has the binary probability of detection without correlations between different coverage units.

The main contributions of this paper are as follows. First, we identify the main limitations of disk coverage model in describing the real-world sensing coverage behaviors. We argue that the gap between the disk model and real sensing is so significant that most of coverage problems may need a revisit in real-world applications. Second, we systematically study the sensing coverage characteristics of a real sensing application. By theoretical and empirical investigations we derive T-R model. The relations between the locations of the target objects, the detection probability, the detection errors and the impacts of some system control parameters are quantitatively given. T-R model is a non-node centric and probabilistic model with correlations among different coverage units. This is, to the best of our knowledge,

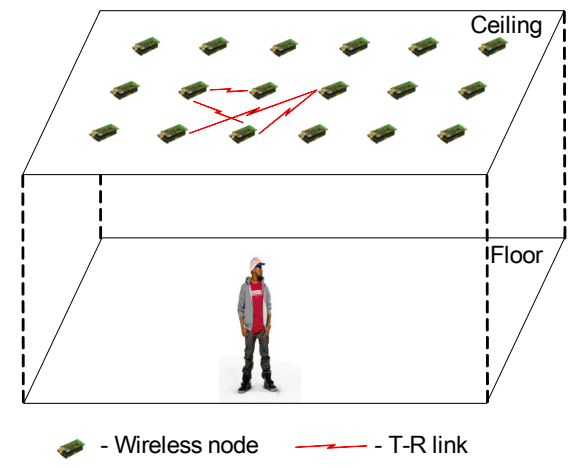

Fig. 2. Illustration of transceiver-free object detection

the first model that includes all these features. Third, we conduct comprehensive field studies to evaluate the performance of T-R model. Test-bed result based on 30 telosB nodes [24] shows that T-R model is more appropriate than the traditional disk model. The average error between the model and the reality is $8 \%$, which is acceptable for most applications.

The rest of this paper is organized as follows. In the next section, we will discuss the related works about traditional disk-based models. Sec. III gives some background information about the T-R model, and Sec. IV will detail the derivation of T-R model. We present the numerical analysis as well as implementation experimental results in Sec. $\mathrm{V}$ for the performance evaluation purposes, and conclude the work in the last section. Some possible future work directions will also be listed in the last section.

\section{RELATED WORKS}

The coverage model is mainly for the study of the coverage problem which is a hot topic in wireless sensor networks. Various kinds of coverage have been studied serving for different applications. These kinds of coverage works can be classified into two categories based on their working space.

For two-dimensional field of interests, the main topics includes the blanket coverage [2] [3], barrier coverage [4] and trap coverage [6]. Blanket coverage is a very traditional coverage problem. Assuming that each sensor (or coverage unit) is able to cover certain area, the blanket coverage looks for the optimal deployment of sensors with the minimal number of sensors while each point can be covered by at least one node. Barrier coverage [4] considers a field of barrier and is interested in the question that whether there exists a path passing through the barrier without overlap the covered area of any node. It is a weaker coverage than the blanket coverage and thus needs a much fewer number of sensors to guarantee. It is very useful for the protection of the international 
boarders from the potential intrusions. Trap coverage [6] allows coverage holes within a certain degree. Coverage with fault-tolerance capability is also studied and the $k$-coverage problems are also extensively studied. For three-dimensional space, 3-D full space coverage [8] is a simple extension of blanket coverage to 3-D space, while the optimal deployment with the minimal number of sensors is an open question for hundreds of years [11] [12]. In the 3-D surface coverage [5], the authors noticed that in practice the field is often a complex surface in which the traditional blanket coverage becomes a NPC problem. Besides these purely sensing coverage studies, another major portion of works jointly considers the sensing coverage and the communication connectivity [2] [3]. They conclude that under different ratio of the sensing range and communication range, the optimal deployment will be fairly different, and some of the optimal deployments are still open and unknown. Though many efforts have been devoted to the coverage related problems in wireless sensor networks, all the works above are based on the simply disk model (in 3-D space it is a ball model). Concerning the huge gap between the disk model and the practice, we believe that their results will be far from expected in real environments and need a revisit for most of them.

Non-disk coverage models have also been studied to some extend. Cao et al. [17] suggested that the sensing range of a passive infrared (PIR) sensor roughly followed a 2-D Gaussian distribution. Loukas Lazos et al. [18] and Miorandi et al. [9] considered a heterogeneous sensing model. The sensing coverage area of a sensor could have any arbitrary shape and could be irregular. Visual sensor networks [14] used camera sensors which have the direction constraints and the sensing coverage area is a sector. Some work [20] put forward a probabilistic model for visual sensor. All these existing non-disk models are, however, still based on the disk model. The coverage areas are still centered at sensors. Many of these problems can be done by extensions of the existing approaches. For instance, An inner disk can be a good approximation for the irregular coverage areas. One particular work using a non-disk, probabilistic model is done by Xing et al [10]. They pointed out that the traditional disk model can hardly describe the sensing behaviors well and applied a node-centric probabilistic coverage model. They assumed that the probabilistic coverage model was available and showed that how to use data-fusion techniques to improve the coverage quality. They did not mention how to obtain the model. Our work is, to some extent, motivated by this work. To our best knowledge, we are the first to systematically study the probabilistic and non-node centric coverage model from real applications.
TABLE I

NOTATIONS USED IN THIS PAPER

\begin{tabular}{|l|l|}
\hline Notation & Description \\
\hline$e=\{u, v\}$ & $\begin{array}{l}e \text { is a T-R link, } u \text { is the transmitter of } \\
\text { link } e, v \text { is the receiver of link } e\end{array}$ \\
\hline$\triangle P$ & Difference of received power \\
\hline$\triangle P$ & $\triangle P$ after normalization \\
\hline$\triangle P_{t h}$ & $\begin{array}{l}\text { Threshold of } \triangle P \text { to determine the pres- } \\
\text { ence of the target }\end{array}$ \\
\hline$P r_{e}(x, y)$ & $\begin{array}{l}\text { The probability that } \triangle P \text { is larger than the } \\
\text { given threshold (object presence) } \triangle P_{t h} \\
\text { for T-R link } e\end{array}$ \\
\hline$P r_{t h}$ & $\begin{array}{l}\text { Threshold of } P r_{e}(x, y) \text { to define the cov- } \\
\text { erage area }\end{array}$ \\
\hline$C(e)$ & $\begin{array}{l}\text { Coverage area of a T-R link } e, C(e)= \\
(x, y) \mid P r_{e}(x, y) \geq P r_{t h}\end{array}$ \\
\hline$P r_{E}(x, y)$ & $\begin{array}{l}\text { The probability when the whole set of } \\
\text { links } E \text { are in present }\end{array}$ \\
\hline$\varepsilon(e, E)$ & $\begin{array}{l}\text { A function to calibrate the detection } \\
\text { probability of } e \text { when the whole set of } \\
\text { links } E \text { are in present }\end{array}$ \\
\hline
\end{tabular}

\section{PRELIMINARY}

In this section, we present the preliminary of the T$\mathrm{R}$ model. We first introduce the transceiver-free object detection which is the application background of the new coverage model. From this application we summarize the main unique characteristics of the individual coverage unit. In the last we will give some examples of the coverage problem under the new model.

\section{A. Application background}

Transceiver-free objects tracking [21] [22] and devicefree passive localization [23] have attracted an increasing attention in recent years. It is mainly designed for security and surveillance, intrusion detection, outdoor asset protection, and location-aware applications. It can locate and track the objects without introducing any intrusion to the target. It therefore can be conducted in an absolutely stealthy manner. The basic idea of transceiverfree detection is to use the disturbance of the target to the proximity radio environment to estimate the presence of the target.

Figure 2 gives an example to illustrate the system architecture. A number of nodes are deployed in advance at the known positions, e.g., the ceiling of an indoor environment. These nodes continuously transmit packets (can be useful or dummy ones) and measure the signal strength of the corresponding links. The target objects will be detected when certain links have significant changes on the signal strength. Such links are called $T$ $R$ links (shorted for Transmitter-Receiver links). In the illustration of Fig. 2, people are the object of interests. When they are presence, additional wireless transmission paths will be introduced between nodes (this is often referred as the multipath effect [26]). As a result, the 


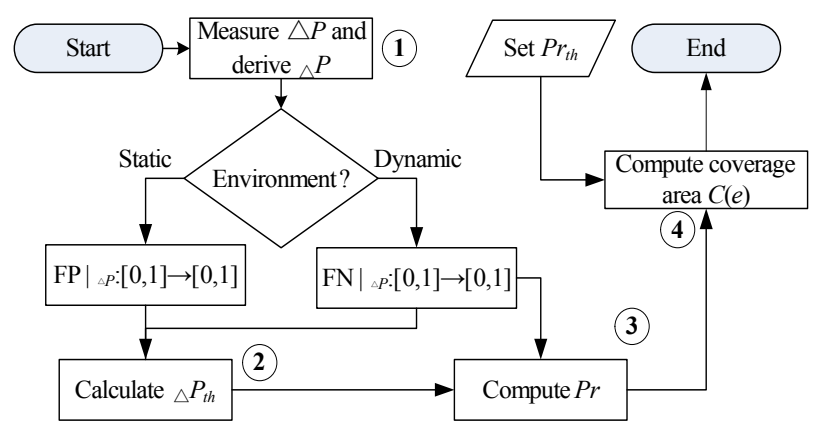

Fig. 3. Flowchart of T-R model derivation

signal strength will be greatly influenced depending on the location of the object. By observing these T-R links we can infer the positions of the target objects. To simplify the presentation, in the rest of this paper we refer an environment with dynamic target objects as dynamic environment and the counterpart as static environment, though in practice we do not know whether an environment is dynamic or static. Actually, to distinguish the dynamic and static environments is just the main objective of the transceiver-free object detection.

In a system of transceiver-free object detection, the T-R links are the basic coverage unit. Obviously their sensing behavior can hardly be described by the deterministic disk model. In the next subsection, we will summarize the main characteristics of the sensing coverage behaviors.

\section{B. Unique features}

In this subsection, we will summarize the main characteristics of the sensing behavior in the transceiver-free object detection application. We focus our attention on the gap between the disk model and the practice.

1) Probabilistic model: In transceiver-free object detection, the wireless signal strength and its measurement are used to accomplish the task. In practice, the measured signal strength is a random variable that depends on many factors such as the transmission power [25], the path fading [26] and the multipath effect. When we use this information to detect the presence of objects, the detection becomes a random process with a probability of detection. As a result, the sensing coverage is also probabilistic.

2) Link centric: In transceiver-free object detection, the basic coverage unit is the T-R links, rather than the individual sensor nodes in traditional models. The coverage area of each coverage unit is centered at the middle point of a T-R link the nodes themselves.

3) Dependence: The measurement of the wireless links signal strength relies on the transmissions between sensor nodes. Typical sensors have only one transceiver

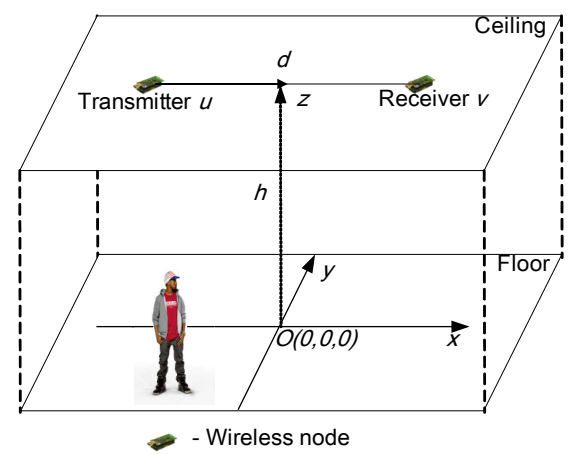

Fig. 4. Coordinate definition

and one antenna, allowing one transmission (either transmitting or receiving) at a time. Moreover, different transmissions may interfere from each other if they are too close. Consequently, there will be various dependencies between different T-R links and their coverage areas. The dependency include: 1) exclusive, such as the case when two T-R links are sharing the same receiver; 2) interfere, when two T-R links are too close to each other, though they share neither transmitter nor receiver; 3) independency, meaning that the two T-R links have no dependency. Recall that in the disk model, all coverage units are assumed to be independent.

4) Shape of the coverage area: The area with the sensing capability that a T-R link can provide depends on the length of the link. Ideally it is like an eclipse but a disk.

\section{Coverage problem under T-R model}

To accurately describe these characteristics of the sensing, we propose the T-R model. With these characteristics and the model, a typical blanket coverage problem then becomes how to guarantee that an object can be detected with a certain probability in a field using a minimal number of sensors. This problem definition is consistence with a recent work and has been addressed by Xing et. al [10]. Other coverage problems can also be simply extended under the new coverage model, while their solutions are still open. In the next, we will theoretically and systematically derive the T-R model to describe these sensing characteristics.

\section{T-R COVERAge Model}

In this section, we will give the detailed derivation of the T-R model. We first present the workflow of the derivation, and then explain the main steps in detail.

\section{A. Workflow of the model derivation}

$\mathrm{T}-\mathrm{R}$ model is a probabilistic model. The major goal of the derivation is to obtain the relation between the detection probability and the location of the target object, 


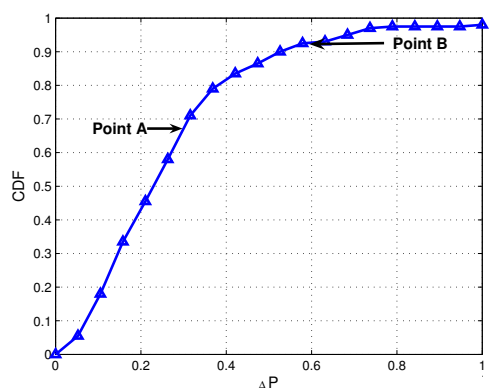

Fig. 5. Theoretical estimation of $\triangle P$ in static environment

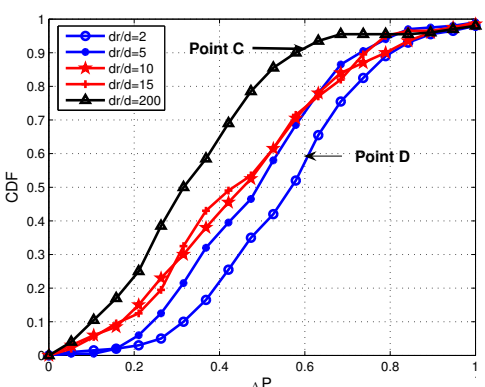

Fig. 6. Theoretical estimation of $\triangle P$ in dynamic environment and based on these relations to give the coverage area of each coverage unit. Figure 3 gives the workflow of the derivation which mainly consists of four procedures as follows.

For each T-R link, we first measure the difference of the received signal strength $\triangle P$ which is the core of the object detection. This measured difference $\triangle P$ is then normalized to $\triangle P$ to be comparable (labeled as a circled 1 in Fig. 3). In the second step, we calculate an appropriate threshold $\triangle P_{t h}$ for $\triangle P$ to strike the tradeoff between different detection errors, i.e., the false positive and false negative (formal definitions will be in later subsection). With the determined threshold $\triangle P_{t h}$ we are able to compute the detection probability $\operatorname{Pr}$ for each target location in the third step. Optionally, we can also compute the coverage area for each coverage unit (T-R link) when a threshold of $\mathrm{Pr}$ is given.

In the next, we will detail these four steps respectively. Some notations that will be used in this paper are listed in Table 1.

\section{B. Measurement of the difference of the signal strength $\triangle P$ and its normalization}

For the measurement, we assume that a present object is always on the ground and a T-R link $\{u, v\}$ is deployed on the ceiling parallel to the ground (Fig. 4). Node $u$ is the transmitter and $v$ is the receiver. To simplify the presentation, suppose there is a $3-\mathrm{D}$ coordinate system according to the $\mathrm{T}-\mathrm{R}$ link $e=\{u, v\}$. The origin of the coordinate system is the mapping of middle point of $\{u, v\}$ on the ground and the $x$-axis is the direction of $\overrightarrow{u v}$. The $y$-axis is then the mapping of the perpendicular line of $\overrightarrow{u v}$. Suppose the height of the ceiling is $h$. Let $d=|\overrightarrow{u v}|$ be the distance between $u$ and $v$. Notice that under this system the node $u$ has the coordinate $u$ : $(-d / 2,0, h)$ and $v$ is: $(d / 2,0, h)$.

In a static environment (no target presence), there are mainly two radio propagation paths between $u$ and $v$. One is the line-of-sight path, and the other is reflected by the ground. To simplify the analysis, ignore the other multi-path reflections.

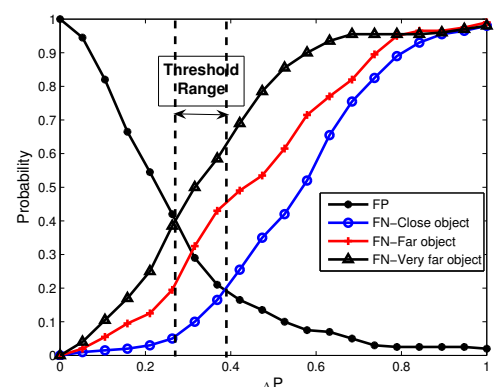

Fig. 7. FP and FN in theory

When the target object is in presence (dynamic environment), the coordinate of the object is denoted as $(x, y, z)^{1}$ This object will cause one additional reflection path. According to the log-distance path loss model [21], the difference of the received power at $v$ is

$$
\triangle P=c_{1}+c_{2} \log _{10}\left(4 x^{2}+d^{2}+4 y^{2}+4 h^{2}\right)+G
$$

where $c_{1}$ and $c_{2}$ are two constants that depend on many environmental factors such as the transmission power and the path loss exponent. The term $G$ is a random variable that can be modeled with Rice distribution [21] with the probability density function

$$
g(\varsigma \mid \gamma, \delta)=\frac{\varsigma}{\delta^{2}} \exp \left(-\frac{\varsigma^{2}+\gamma^{2}}{2 \delta^{2}}\right) I_{0}\left(\frac{\varsigma \gamma}{\delta^{2}}\right)
$$

where $\gamma$ is the amplitude of the disturbed signal, and $\delta$ is the standard deviation of the Gaussian noise source. This distribution is uniquely defined by $\frac{\gamma^{2}}{2 \delta^{2}}$, which is the ratio of the signal power in the dominant line-of-sight signal power over the scattered signal power. Rice distribution [28] is usually applied when there is a dominating lineof-sight signal. Eq. 1 can be rewritten as $\triangle P=f(x, y)+$ $G$ where

$$
f(x, y)=c_{1}+c_{2} \log _{10}\left(4 x^{2}+d^{2}+4 y^{2}+4 h^{2}\right)
$$

In practice, we use a normalized value $\triangle P$ so that the detections of objects in different locations are comparable. This normalized value $\triangle P$ is

$$
{ }_{\triangle} P=\frac{\triangle P(x, y)-\min (\triangle P(x, y))}{\max (\triangle P(x, y))-\min (\triangle P(x, y))}
$$

where $\max (\triangle P(x, y))$ and $\min (\triangle P(x, y))$ are the maximum and minimum values of $\triangle P$. They can be estimated by calculating the statistics of a large number of $\triangle P$ samples, e.g., 1000 . In the remainder of this paper, we will use $\triangle P$ as the main measurements of the object presence.

\footnotetext{
${ }^{1}$ We assume the targets are always on the ground, therefore the coordinate will be $(x, y, 0)$, or shorted as $(x, y)$ if there is no confusion
} 


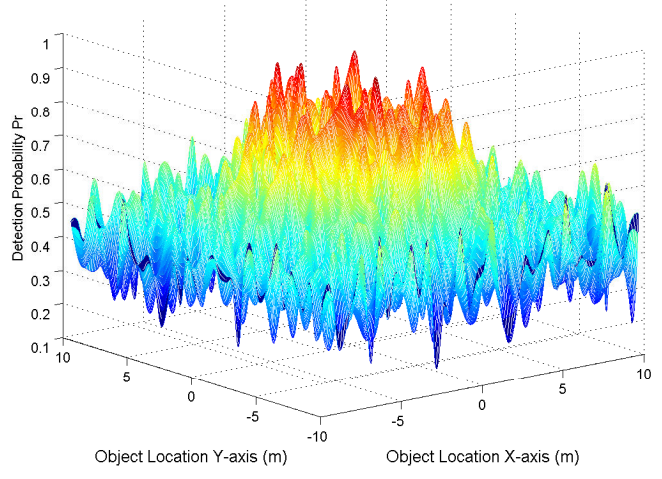

Fig. 8. Object detection probability by T-R model

Equation 1 shows that, in general, when the target object is far away from the center point of the coordinates (point $O$ in Fig. 4), $\triangle P$ is smaller, and vice versa. In practice, we set a threshold $\triangle P_{t h}$ to determine the presence of the target. When $\triangle P \geq \triangle P_{t h}$, we claim the presence of an object, or no presence if otherwise. Notice that $\triangle P$ is a random variable and cannot be precisely measured. Therefore detection errors are inevitable. In the next we discuss the relation between these detection errors and $\triangle P_{t h}$.

\section{Determination of the threshold $\triangle P_{t h}$}

The threshold $\triangle P_{t h}$ is critical for the object detection errors. Basically, there are two kinds of errors. One is False Positive (FP) and the other one is False Negative (FN). Both of them are highly related on the $\triangle P$. In this subsection we study these relations respectively.

Definition False Positive (FP) is the probability that there is no target object in the field of interest (static environment), while the detection reports the presence.

FP occurs in the static environment while no object is presence. We set $d_{r} \rightarrow \infty$ in Eq.1 to represent the static environment (i.e., the object is infinitely far). Notice that even under static environments, there are still received power variance. We sample $100 \triangle P$ and their Cumulative Distribution Function (CDF) is shown in Figure 5. In general, a smaller threshold of $\triangle P$ will introduce more FP and vice verse. For instance, when $\triangle P_{t h}=0.6$, FP is about 0.1 (Point $B$ in Fig. 5). And when $\triangle P_{t h}$ is 0.3 , FP increases to 0.32 (Point $A$ in Fig. 5).

Definition False Negative (FN) is the probability that the target object is in the field of interest (dynamic environment), while the detection fails to report it.

FN exists in the dynamic environment where the target object is presence. We apply Eq. 1 to model the dynamic environment. For each object position, we sample 100

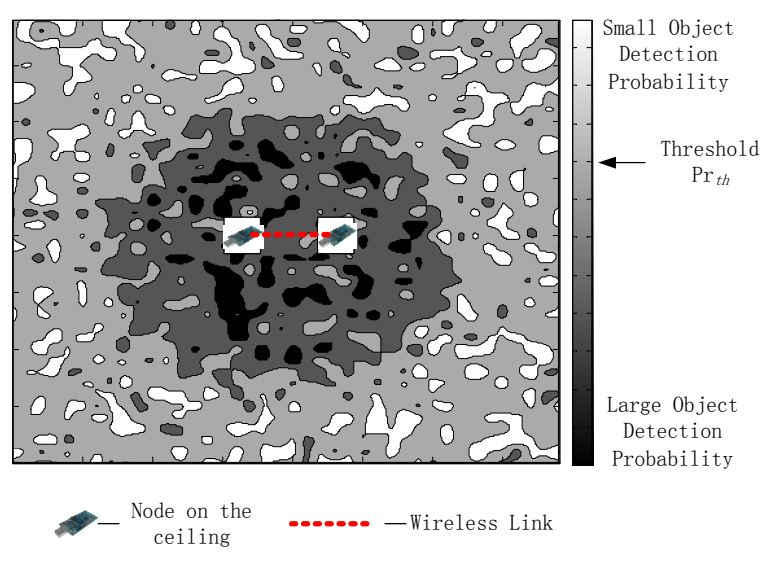

Fig. 9. Coverage area of individual link

$\triangle P$ and depict the CDF in Fig. 6. The different plots are for object in different positions. The different positions are represented by the ratio between the length of the new added path and that of the line-of-sight path. A larger ratio indicates a farther object from the T-R link. To the opposite of FP, a higher $\triangle P_{t h}$ will introduce more $\mathrm{FN}$ and vice verse. For instance $\triangle P_{t h}=0.6$ makes $\mathrm{FN}=0.91$ (Point $C$ in Fig. 6) for a farther node (the ratio=200). For the close object, FN is 0.59 under the same $\triangle P_{t h}$ (Point $D$ in Fig. 6). If we set a lower $\triangle P_{t h}$, the FN will decrease too.

According to these results, there is a nature tradeoff between FP and FN. Figure 7 depicts both FP and FN in a single figure. It shows that a lower threshold will have a lower FN but more FP, and vice verse. Such a tradeoff is natural as it is widely accepted that FP and FN have the intrinsic tradeoff in any decision making systems. The setting of $\triangle P_{t h}$ is the striking of such a tradeoff. A feasible $\triangle P_{t h}$ is the one that minimizes the summation of the FP and FN, i.e.,

$$
{ }_{\triangle} P_{t h}=\arg \min _{\triangle P}(F N(\triangle P)+F P(\triangle P))
$$

where $\operatorname{FP}(\triangle P)$ is the function the expresses the relation between FP and $\triangle P$, and $\mathrm{FN}(\triangle P)$ is that for $\mathrm{FN}$. Notice that the function $\operatorname{FP}(\triangle P)$ is independent to the target object while $\mathrm{FN}(\triangle P)$ depends on the position of the objects. Nevertheless, the impacts of the positions are insignificance. For instance, for distant object (i.e., the ratio=200) the $\triangle P_{t h}$ is 0.28 and for a nearby object is 0.39 . In practice, thresholds between 0.28 and 0.39 are suggested.

\section{T-R coverage model for individual link}

With the $\triangle P_{t h}$ being appropriately set, we are able to derive the object detection probability. Given an individual T-R link $e=\{u, v\}$, the object detection probability at a location $(x, y)$, denoted as $\operatorname{Pr}_{e}(x, y)$, is 


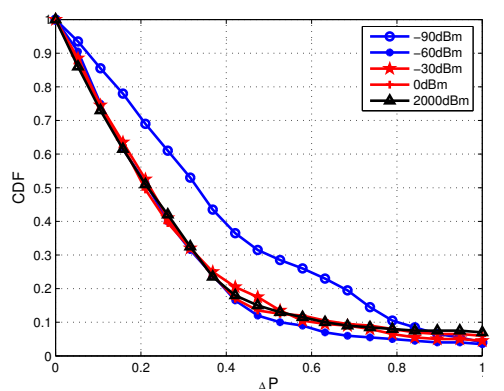

Fig. 10. Impact of different transmission power on FP

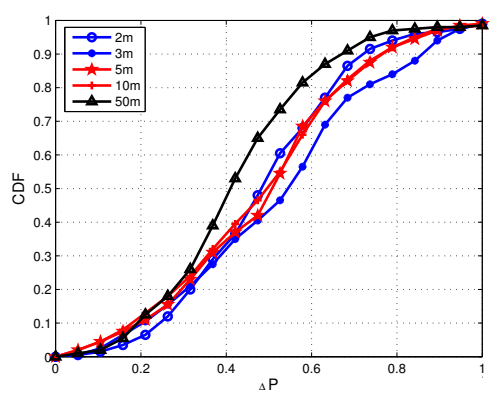

Fig. 13. Impact of node distance on FN

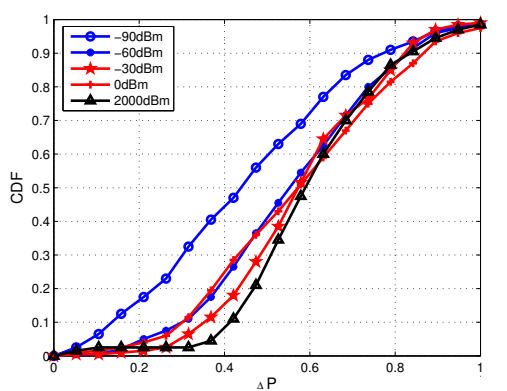

Fig. 11. Impact of different transmission power on FN

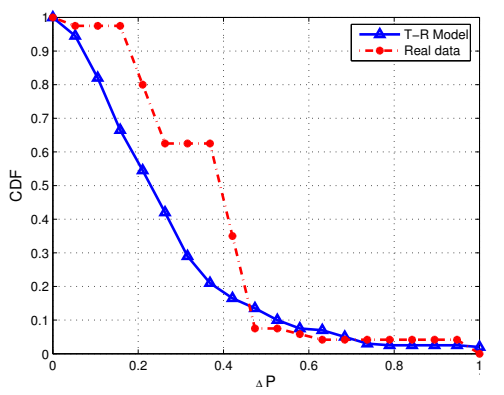

Fig. 14. FP comparison in static environment

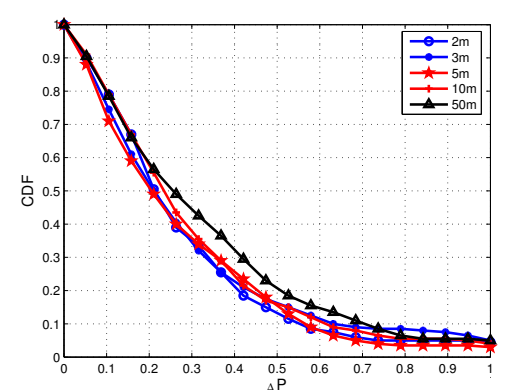

Fig. 12. Impact of node distance on FP

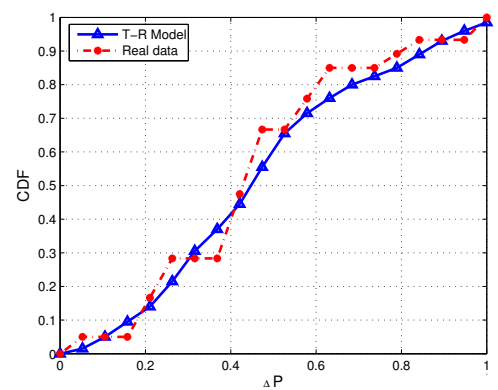

Fig. 15. FN comparison for nearby object the probability that $\triangle P$ is larger than a given threshold $\triangle P_{t h}$, i.e.,

$$
\operatorname{Pr}_{e}(x, y)=\operatorname{Pr}_{e}\left(\triangle P(x, y) \geq_{\triangle} P_{t h}\right)
$$

Figure 8 shows $\operatorname{Pr}_{e}(x, y)$ at a field of $20 m \times 20 m$ (it is a reasonably large area for the detection of a single link in transceiver-free object detection). We set $\triangle P_{t h}=0.3$ according to the suggestions. The height of the ceiling is set to be typical as $2.4 \mathrm{~m}$. The length of the T-R link is $3 m$ and thus the coordinates of the transmitter and receiver are $(-1.5,0,2.4)$ and $(-1.5,0,2.4)$. Not surprising, the detection probability is high when the target is close to the T-R link, and small when otherwise.

Through with a given T-R link any location in the field has certain detection probability, from the coverage perspective it makes less sense to claim that the location is covered when the detection probability is extremely low. For this we use a threshold $P r_{t h}$ to ignore the locations of low detection probabilities. It is set based on the application requirements. Given a coverage unit T-R link, a location is said to be covered if its detection probability is greater than the given threshold $P r_{t h}$. Accordingly, the coverage area of a T-R link $e$, denoted as $C(e)$, is the set of locations that have the detection probability being greater than the threshold $\mathrm{Pr}_{t h}$, i.e.,

$$
C(e)=(x, y) \mid P r_{e}(x, y) \geq P r_{t h}
$$

Fig. 9 illustrates an example of $C(e)$ in a bird's-eye view. The coverage areas of different $P r_{t h}$ are depicted with different gray values. From the results we can find that the coverage area of a T-R link is roughly an ellipse with a fluctuated border. Its size depends on the setting of the threshold $\operatorname{Pr}_{t h}$.

\section{E. T-R coverage model for multiple links}

A unique feature of T-R model is that different coverage units (T-R links) are no further independent and their coverage as well. In this subsection, we derive the correlations between the coverage of different T-R links.

In general, more T-R links simultaneously residing in the field cause a higher difference of the received power $\triangle P$, and therefore more detection probabilities. Suppose we have a set of T-R links $E$ simultaneously detecting a location $(x, y)$. And any T-R link has the individual detection probability $\operatorname{Pr}_{e}(x, y)$. The accumulated detection probability by this set of links can be obtained by inclusive-exclusive principles in probability theory [27], i.e.,

$$
\operatorname{Pr}_{E}(x, y)=1-\prod_{e \in E}\left(1-\varepsilon(e, E) \cdot \operatorname{Pr}_{e}(x, y)\right)
$$

where $\varepsilon(e, E)$ is a function to calibrate the detection probability of $e$ when the whole set of links $E$ are in present. Notice that in practice this calibration function $\varepsilon(e, E)$ depends on the interferences among different links. It is difficult, if not impossible, to be accurately obtained. In this work we use some empirical measurements to approximate it. 


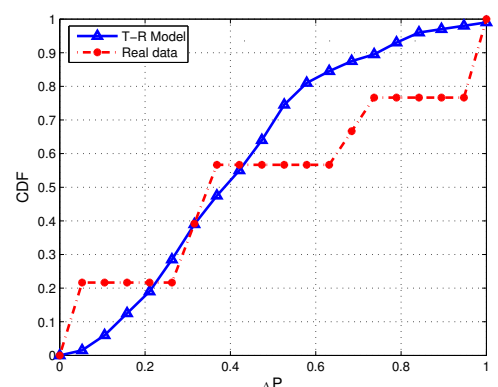

Fig. 16. FN comparison for distant object

\section{EXPERIMENT RESULTS}

In this section, we evaluate the performance of $\mathrm{T}$ $\mathrm{R}$ model through theoretical analysis and implementation experiments. We first study the impacts of key parameters in T-R model on FN and FP in detection using numerical analysis. We then evaluate the T-R model for individual T-R link based on implementation experiments, and the T-R model for multiple T-R links in the last.

\section{A. Impact of transmission power on FP and FN}

Transmission power is a key parameter on the performance of the object detection, and therefore it has the critical impacts on the T-R model. We depict FP and FN with varying transmission power $T x$. from $-90 \mathrm{dBm}$ to $200 \mathrm{dBm}$. At each $T x$. power level, $100 \triangle P$ samples are generated and the results are shown in Fig. 10 (FP in static) and Fig. 11 (FN in dynamic). According to these results, we can find that the $\mathrm{FP}$ and $\mathrm{FN}$ are not sensitive to the transmission power $T x$. unless it is very low. The plots of FP under different $T x$. are very close to each other when $T x$. $\geq-60 \mathrm{dBm}$. The plots of FN have a similar observation. Therefore, the detection probability $\operatorname{Pr}$ for different links will be almost the same for a given threshold $\mathrm{Pr}_{t h}$, which is a very promising feature for the coverage problem study.

\section{B. Impact of node distance}

Another important factor in T-R model is the distance between the transmitter and receiver of the T-R link. In this set of experiments we study this impact by varying the distance in between from $2 \mathrm{~m}$ to $50 \mathrm{~m}$. The other parameters are kept fixed. Fig. 12 and Fig. 13 show the resulted in FP and FN under these scenarios. From these results we can find that the impact is also insignificance when the distance is no more than $10 \mathrm{~m}$. The averaged difference between the farthest distance $10 \mathrm{~m}$ and the closet one $2 m$ is only about $5 \%$. Accordingly, the detection probability can also be considered as a constant when the distance between the transmitter and receiver is not too large (i.e., less than $10 \mathrm{~m}$ ).

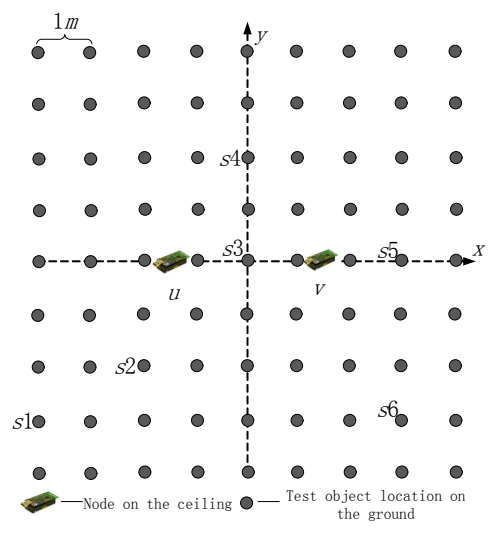

Fig. 17. Test locations

\section{Verification of individual T-R coverage model}

In this subsection, we use real measurements to evaluate the performance of the T-R model for individual T$\mathrm{R}$ links. We conduct the implementation measurements in a mid-sized laboratory of $12 \times 8 \mathrm{~m}^{2}$. We hang two telosB [24] sensor nodes on the ceiling to function as the transmitter and receiver. They are $3 \mathrm{~m}$ apart and the height of the ceiling is $2.4 \mathrm{~m}$. We use $2.4 G H z$ ISM band and conduct the experiments in a radio clean environment. The transmission power is set as $0 d B m$. For static environment, we measure the received power, compute the FP and compare it with that of T-R model. As shown in Fig. 14, T-R model can accurately approximate the real detection performance for the static environment. We conduct 50 independent runs and the average error between the real FP and the FP by model is only $15 \%$ in average.

For dynamic environment we use a person to function as the target object. The person stands on different locations nearby the deployed T-R link and we collect statistics for each location. For each run, 100 samples are collected for each depicted FN and 100 independent runs are conducted for each location. Among all these results, two representative ones are depicted. Fig. 15 shows the FN of detection for a nearby object and Fig. 16 is for a distant object. Experimental results show that, T-R model can also accurately describe the sensing behavior of dynamic environments. The averaged error between the real FN and FN by T-R model is only $13 \%$.

To evaluate the performance of T-R model in terms of the detection probability, we conduct further experiments. We set a typical detection probability threshold $P r_{t h}=0.30$ and measure the real detection probability at different locations. The locations in this set of experiments are illustrated in Fig. 17 and the results of six representative locations are depicted in Fig. 18. From the results we can find that the T-R model is also promising in terms of the detection probability. For 


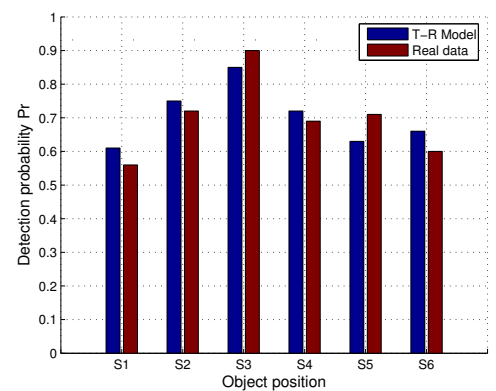

Fig. 18. Detection probability comparison

these six locations, the maximal error is only $9 \%$ and the minimal is only $5.5 \%$. Indeed, for all the 81 locations in the coverage area, the averaged error of the detection probability by T-R model is only $8 \%$. The results for other object locations are omitted due to space limitation.

\section{$D$. Verification of multiple T-R coverage model}

In this part, we evaluate $T-R$ model for multiple $T-R$ links. We investigate the error of T-R model with two T$\mathrm{R}$ links and leave the scenarios of more links for future work. Besides the original T-R link $e=\{u, v\}$, one additional T-R link $e^{\prime}=\left\{u^{\prime}, v^{\prime}\right\}$ is deployed to the field, as shown in Fig. 19. The new deployed link $e^{\prime}$ is parallel to link $e$. The distance of these two links is set as $3 \mathrm{~m}$. Except the location, $u^{\prime}$ and $v^{\prime}$ have the same parameter setting as $u$ and $v$, respectively. Under such scenarios, the T-R links $\{u, v\}$ and $\left\{u^{\prime}, v^{\prime}\right\}$ will have the interference relation. The FP and FN before and after the addition of link $e^{\prime}$ at the location $s 3$ are plotted in Fig. 20 and Fig. 21 respectively.

In general, interference will introduce more detection errors. Both FP and FN increases after link $e^{\prime}$ is introduced. Moreover, we see more impacts of the interference on FP than on FN. For FP, the difference between the scenarios of before and after addition $e^{\prime}$ is more than $15 \%$, and that for $\mathrm{FN}$ is no more than $10 \%$.

The interference on different links will cause higher FP and higher FN, resulting in a lower object detection probability. For instance, we depict FP and FN of location $s 1$ in Fig. 22. Before the interference, the average detection probability is about $56 \%$ and with the interference, it is reduced to $50 \%$. Let whole T-R link set $E=\left\{e, e^{\prime}\right\}$. In this case, an appropriate $\varepsilon(e, E)$ is $0.5 / 0.56=0.89$, though in practice, this may be hard to obtain.

\section{Vi. CONClusion And Future Work}

In this paper, we propose a novel coverage model called T-R model. It is able to fill the gap between the traditional disk model and the real-world sensing behaviors. Though it is derived from a particular application

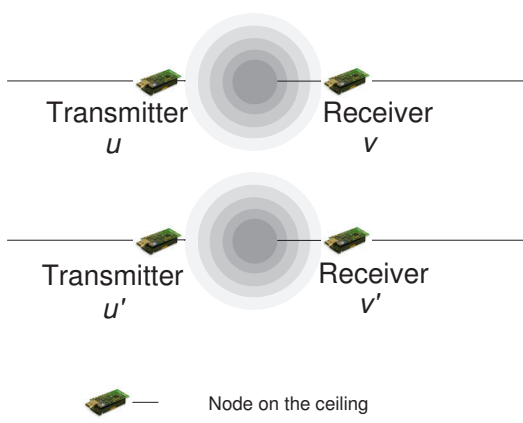

Fig. 19. Scenario of multiple T-R links

of transceiver-free object tracking, it presents promising generality which enable it be applied in a much broader scope of application. With this new coverage model, many of the existing coverage problems such as the blanket coverage, barrier coverage and trap coverage may need a revisit.

$\mathrm{T}-\mathrm{R}$ model is a link-centric probabilistic model derived from the transceiver-free object detection. We found that like other decision making systems, in T-R model there is also a nature tradeoff between the False Negative (FN) and False Positive (FP). Based on these two errors the coverage area of a coverage unit can be determined. We use implementation experiments to evaluate the performance of T-R model and the results are fair promising. The error between the model and the reality is around $20 \%$ and as low as $5 \%$ in some cases. We also have the surprising observations that some key control parameters in device free object detection (e.g., the transmission power and distance between nodes) do not have the significant impacts on the accuracy of the model. In other words, T-R model is, to some extent, less dependent on the system configuration. This allows T-R model that derived from the specific applications to be applied in a more general way.

The future work can be conducted along following directions. First, based on this new model, some fundamental coverage problem becomes open. For instance, the traditional blanket coverage becomes to look for the minimal number of sensors and the deployment so that the detection probability of every location in the field is more than a given threshold. Second, for T-R model of multiple links, we only conduct experiments of three nodes and two links, and more are left for the next step work. Moreover, some important parameters are still obtained by empirical studies, e.g., the correlation function $\varepsilon(e, E)$. Its theoretical foundation is calling for further investigations.

\section{ACKNOWLEDGE}

This research was supported in part by Hong Kong RGC Grant HKUST617908, China NSFC Grant 


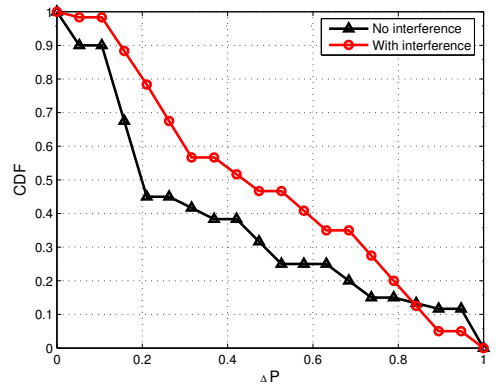

Fig. 20. FP comparison of T-R link $u v$ before and after interference

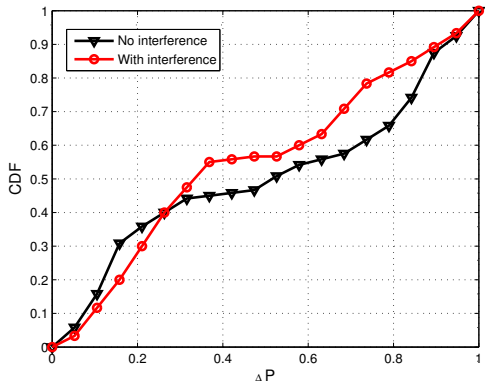

Fig. 21. FN comparison of T-R link $u v$ before and after interference

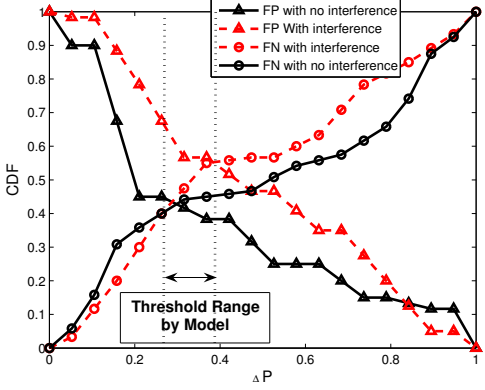

Fig. 22. Comparison of FP and FN
60933011, the National Basic Research Program of China (973 Program) under Grant No. 2006CB303000, the National Hi-Tech R\&D Program of China (863 Program) under Grant No. 2008AA01A324, the National Science and Technology Major Project of China under Grant No. 2009ZX03006 - 001, and the Science and Technology Planning Project of Guangdong Province, China under Grant No. 2009A080207002.

\section{REFERENCES}

[1] S. Meguerdichian, F. Koushanfar, M. Potkonjak and M. B. Srivastava, "Coverage Problems in Wireless Ad-hoc Sensor Networks", in Proceeding of the IEEE Conference on Computer Communications (INFOCOM), 2001.

[2] X. Bai, S. Kumar, D. Xuan, Z. Yun and T. H. Lai,"Deploying Wireless Sensors to Achieve Both Coverage and Connectivity", in Proceeding of ACM International Symposium on Mobile Ad Hoc Networking and Computing (MobiHoc), 2006.

[3] X. Bai, Z. Yun, D. Xuan, T. H. Lai and W. Jia, "Deploying FourConnectivity And Full-Coverage Wireless Sensor Networks", in Proceeding of the IEEE Conference on Computer Communications (INFOCOM), 2008.

[4] S. Kumar, T. H. Lai and A. Arora, "Barrier Coverage With Wireless Sensors", in Proceedings of ACM International Conference on Mobile Computing and Networking (MobiCom), 2005.

[5] M. Zhao, J. Lei, M. Wu, Y. Liu and W. Shu, "Surface Coverage in Wireless Sensor Networks", in Proceeding of the IEEE Conference on Computer Communications (INFOCOM),2009.

[6] P. Balister, Z. Zheng, S. Kumar and P. Sinha, "Trap Coverage: Allowing Coverage Holes of Bounded Diameter in Wireless Sensor Networks", in Proceeding of the IEEE Conference on Computer Communications (INFOCOM), 2009.

[7] X. Bai, C. Zhang, D. Xuan, J. Teng, W. Jia, "Low-Connectivity and Full-Coverage Three Dimensional Networks", in Proceeding of ACM International Symposium on Mobile Ad Hoc Networking and Computing (MobiHoc), 2006.

[8] S. M. Nazrul Alam and Zygmunt J. Haas, "Coverage and Connectivity in Three-Dimensional Networks", in Proceedings of ACM International Conference on Mobile Computing and Networking (MobiCom), 2006.

[9] D. Miorandi and E. Altman, "Coverage and connectivity of ad hoc networks", in Proceeding of the IEEE Conference on Computer Communications (INFOCOM), 2005.

[10] G. Xing, R. Tan, B. Liu, J. Wang, X. Jia and C. Yi, "Data Fusion Improves the Coverage of Wireless Sensor Networks", in Proceedings of ACM International Conference on Mobile Computing and Networking (MobiCom), 2009.

[11] R. P. Bambah, "On lattice coverings by spheres", in National Science, no. 10, pp. 25-52, 1954.

[12] Thomson and S. W. L. Kelvin, "On the division of spacewith minimum partition area", in Philosophical Magazine no. 24, pp. 503-514, 1887.
[13] R. Iyengar, K. Kar and S. Banerjee, "Low-coordination Topologies for Redundancy in Sensor Networks", in Proceeding of ACM International Symposium on Mobile Ad Hoc Networking and Computing (MobiHoc), 2005.

[14] W. Shaw, Y. He and I. Lee, "Mobile Sink to Track Multiple Targets in Wireless Visual Sensor Networks", in Proceedings of IEEE Ubiquitous Multimedia Computing (UMC), 2008.

[15] P. Bahl and V. N. Padmanabhan, "RADAR: An In-Building RFbased User Location and Tracking System", in Proceeding of the IEEE Conference on Computer Communications (INFOCOM), 2000.

[16] A. Arora, et. al, "ExScal: Elements of an Extreme Scale Wireless Sensor Network", in Proceedings of IEEE International Conference on Embedded and Real-Time Computing Systems and Applications (RTCSA), 2005.

[17] Q. Cao, T. Yan, J. A. Stankovic, and T. F. Abdelzaher, "Analysis of Target Detection Performance for Wireless Sensor Networks", in Proceedings of IEEE International Conference on Distributed Computing Systems (ICDCS), 2005.

[18] L. Lazos and R. Poovendran, "Stochastic coverage in heterogeneous sensor networks", in ACM Transaction on Sensor Networks (TOSN), 2(3):325-358, 2006.

[19] R. Szewczyk, A. Mainwaring, J. Polastre, J. Anderson and D. Culler, "An Analysis of a Large Scale Habitat Monitoring Application", in Proceedings of ACM Conference on Embedded Networked Sensor Systems (SenSys), 2004.

[20] M. Fichtner, and A.1 Grobmann, "A Probabilistic Visual Sensor Model for Mobile Robot Localisation in Structured Environments", in Proceedings of IEEE International Conference on Intelligent Robots and Systems (IROS), 2004.

[21] D. Zhang, J. Ma, Q. Chen, and L. M. Ni , "An RF-based system for tracking transceiver-free objects", in Proceeding of IEEE International Conference on Pervasive Computing and Communications (PerCom), 2007.

[22] D. Zhang, L. M. Ni,"Dynamic Clustering for tracking multiple transceiver-free objects", in Proceeding of IEEE International Conference on Pervasive Computing and Communications (PerCom), 2009.

[23] M. Youssef, M. Mah and A. Agrawala, "Challenges: device-free passive localization for wireless environments", in Proceedings of ACM International Conference on Mobile Computing and Networking (MobiCom), 2007.

[24] XBOW Corporation, "TelosB mote specifications" http://www.xbow.com/Products/productdetails.aspx?sid=252.

[25] D. Pozar, "Microwave engineering", in 2nd ed.,John Wiley and Sons, Inc., 1998.

[26] T. S. Rappaport, "Wireless communications principles and practices", in Prentice-Hall, 2002.

[27] G. B. Thomas, M. D. Weir, J. Hass and F. R. Giodano, "Thomas' Calculus", in 11nd ed., Addison Wesley, Inc., 2004.

[28] Abdi, A., Tepedelenlioglu, C., Kaveh, M., and Giannakis, G, "On the estimation of the $\mathrm{K}$ parameter for the Rice fading distribution", in IEEE Communications Letters, vol.5, no.3, pp. 92-94, 2001. 